\title{
Differentially expressed genes in association with in vitro invasiveness of human epithelioid sarcoma
}

\author{
A Weber, R Engers, S Nockemann, L L Gohr, A Zur Hausen, H E Gabbert
}

\begin{abstract}
Aims-Differential display reverse transcription polymerase chain reaction (RTPCR) was performed to identify genes associated with the invasive potential of human epithelioid sarcoma.

Methods-Two different clonal subpopulations, GRU-1A and GRU-1B, derived from the same human epithelioid sarcoma cell line GRU-1 and known to differ greatly in their invasive potential were compared by means of mRNA fingerprinting.

Results-Using a set of 10 arbitrary upstream primers and nine anchored oligo-dT primers, 22 candidate gene fragments were identified; differential expression was confirmed in four of these fragments by northern blot analysis. At the mRNA level, apoferritin light chain was predominantly expressed by the highly invasive cell line GRU-1A. In contrast, the mitochondrial gene M1, encoding cytochrome c oxidase $I$, and the TI-227H gene were expressed more strongly by the low invasive cell line GRU1B. Furthermore, a novel gene fragment was identified and cloned that was preferentially expressed in the low invasive cell line GRU-1B, and therefore might have an inhibitory role in invasion. Consequently, this gene fragment was designated as expressed in low invasive sarcoma cells (ELISC-1).

Conclusions-A novel gene fragment (ELISC-1) and three known genes were identified as potential regulators of tumour invasiveness. Cloning of the entire sequence of ELISC-1 and subsequent investigations are required to establish its biological role.

(F Clin Pathol: Mol Pathol 2001;54:324-330)
\end{abstract}

Institute of Pathology, Heinrich-Heine-

University, Moorenstr.

$5, \mathrm{D}-40225$

Duesseldorf, Germany

$\mathrm{R}$ Engers

S Nockemann

L L Gohr

H E Gabbert

A Weber

A Zur Hausen

Correspondence to:

Dr Engers

engers@

med.uni-duesseldorf.de

Accepted for publication 17 April 2001 actively infiltrate the surrounding tissue. ${ }^{1}$ However, malignant tumour cells arising from the same original tumour often show a broad heterogeneity with respect to both morphology and biological properties, such as invasion and metastasis. This intratumoral heterogeneity probably reflects different steps of progression of an individual tumour cell from a normal towards a malignant invasive and metastatic phenotype. $^{3-5}$ These differences are probably parallelled by differences in the repertoire of genes expressed at a given time.

Recently, we successfully established an in vitro model for studying the mechanisms involved in the regulation of tumour cell invasiveness. The GRU-1 cell line was isolated from a human epithelioid sarcoma, which is a highly malignant soft tissue tumour, typically arising in the extremities of young adults and presenting an extremely aggressive clinical course with frequent metastasis and treatment failure. ${ }^{6}$ Subsequently, the clonal subpopulations GRU-1A and GRU-1B were isolated from GRU-1 and characterised with respect to morphological features, growth kinetics, drug sensitivities, cell motility, cell substrate adhesion, and in vitro invasiveness. ${ }^{7-9}$ In these studies, GRU-1A had a significantly higher invasive potential than GRU-1B. ${ }^{7}$ Because GRU-1A and GRU-1B cells have the same genetic background, but differ with respect to their invasive behaviour, differences in the pattern of gene expression should be responsible for the phenotypic diversity of both clonal cell lines. ${ }^{10}$ Hence, GRU-1A and GRU-1B provide a valuable system for the identification of candidate invasion related genes.

Differential display reverse transcription polymerase chain reaction (RT-PCR), originally introduced by Liang and Pardee in $1992,{ }^{11}$ provides a useful tool for identifying differences in gene expression between related cell populations, and has been applied successfully in an increasing number of studies investigating the molecular nature of cancer. ${ }^{12}{ }^{13} \mathrm{~A}$ comparative analysis has shown that differential display RT-PCR has many advantages (identification of low abundant mRNA, need for only small amounts of starting RNA, and ability to identify increases and decrease in messages) compared with related techniques, such as electronic subtraction or subtractive hybridisation. ${ }^{14}$

In our present study, differential display RT-PCR was used to identify and isolate genes whose expression is associated with the invasive phenotype of two different clonal human sarcoma cell lines. At the RNA level, four different genes were found to be differentially expressed between the two cell lines. Apoferritin light chain was predominantly expressed by the highly invasive cell line GRU-1A, whereas cytochrome c oxidase I, TI-227H, and a novel 
gene fragment (ELISC-1) were more strongly expressed by the low invasive cell line GRU$1 \mathrm{~B}$.

\section{Methods}

CELL LINES AND CELL CULTURE

The clonal subpopulations GRU-1A and GRU-1B were isolated from the human epithelioid sarcoma cell line GRU-1 and have been characterised with respect to morphological features, proliferation kinetics, drug sensitivities, cell motility, cell-substrate adhesion, and in vitro invasiveness. ${ }^{7-9}$ All cell lines were maintained in Dulbecco's modified Eagle medium, supplemented with $10 \%$ fetal calf serum (both Gibco, Karlsruhe, Germany) and antibiotics. Cells were cultured at $37^{\circ} \mathrm{C}$ in an atmosphere of $5 \% \mathrm{CO}_{2}$.

DIFFERENTIAL CDNA DISPLAY

Total RNA was isolated from cells using the Qiagen RNAeasy kit according to the manufacturer's protocol (Qiagen, Hilden, Germany). Differential display was performed by Delta ${ }^{\mathrm{TM}}$ RNA fingerprinting (Clontech Laboratories, Palo Alto, California, USA), essentially as described but with some modifications. Briefly, first strand synthesis was carried out with Moloney murine leukaemia virus (MMLV) reverse transcriptase using $2 \mu \mathrm{g}$ of total RNA from both cell lines. PCR fingerprinting reactions were performed in a Perkin-Elmer Thermal Cycler 2400 (Perkin-Elmer, Norwalk, Connecticut, USA) using pairwise combinations of 10 arbitrary primers ( 25 mer) and nine anchored oligo-dT primers (30 mer). Cycling parameters were as follows: $94^{\circ} \mathrm{C}$ for five minutes, $40^{\circ} \mathrm{C}$ for five minutes, $68^{\circ} \mathrm{C}$ for five minutes for one cycle; $94^{\circ} \mathrm{C}$ for two minutes, $40^{\circ} \mathrm{C}$ for five minutes, $68^{\circ} \mathrm{C}$ for five minutes for two cycles; $94^{\circ} \mathrm{C}$ for one minute, $60^{\circ} \mathrm{C}$ for one minute, $68^{\circ} \mathrm{C}$ for two minutes for 25 cycles; followed by $68^{\circ} \mathrm{C}$ for an additional seven minutes. PCR products were run on a denaturing $6 \%$ polyacrylamide gel (SequaGel- $6^{\mathrm{TM}} ; \mathrm{Bi}-$ ozym, Oldendorf, Germany) and visualised by standard silver staining. ${ }^{15}$ Bands representing potentially differentially expressed mRNAs were excised from the gel, eluted in $01 \times \mathrm{TE}$, and reamplified using the same primers as for the first amplification under the following conditions: $94^{\circ} \mathrm{C}$ for two minutes; $94^{\circ} \mathrm{C}$ for one minute, $60^{\circ} \mathrm{C}$ for one minute, $68^{\circ} \mathrm{C}$ for two minutes for 20 cycles; followed by $68^{\circ} \mathrm{C}$ for an additional seven minutes. Subsequently, PCR products were purified for cloning procedures.

\section{MOLECULAR CLONING}

Reamplified PCR products were subcloned into the pGEM-T vector system (Promega, Madison, Wisconsin, USA). To obtain a representative selection of clones for each band, at least eight white colonies were picked, and inserts were sequence analysed using plasmid specific primers (SP6 and T7) in an ABI 310 automated sequencer (Perkin-Elmer Applied Biosystems, Foster City, California, USA). A computer search for homology was performed against NCBI GenBank data bases.
NORTHERN BLOT ANALYSIS

Total RNA was isolated as described above. Aliquots of $15 \mu \mathrm{g}$ of total RNA were separated on a $1 \%$ agarose formaldehyde gel and blotted on to Hybond $\mathrm{N}$ nylon filters (Amersham, Little Chalfont, Buckinghamshire, UK). DNA probes of independent clones were generated by PCR from the respective cloned plasmid and radiolabelled by random sequence priming, ${ }^{16}{ }^{17}$ using the rediprime ${ }^{\mathrm{TM}}$ DNA labelling system (Amersham). Equal loading was confirmed by methylene blue staining of the membrane and hybridisation with a glyceraldehyde 3-phosphate dehydrogenase (GAPDH) probe. Membranes were prehybridised at $65^{\circ} \mathrm{C}$ in a solution containing $0.5 \mathrm{M} \mathrm{NaPi}(\mathrm{pH} 7.2), 7 \%$ sodium dodecyl sulphate (SDS), and $1 \mathrm{mM}$ EDTA ( $\mathrm{pH}$ 8.0) and hybridised with labelled probes in the same buffer at $65^{\circ} \mathrm{C}$ overnight. Blots were washed to a final stringency of $0.1 \times$ saline sodium citrate (SSC), $0.1 \%$ SDS at $42^{\circ} \mathrm{C}$, and exposed for four to 48 hours at $-80^{\circ} \mathrm{C}$ on to a Kodak X-Omat film (Eastman Kodak, Rochester, New York, USA) with an intensifying screen. The mRNA expression levels of at least two independent experiments were measured by densitometric analysis, as described previously. ${ }^{7}$

\section{SOUTHERN BLOT ANALYSIS}

Southern blot analysis was performed for those probes for which we were unable to obtain a signal by northern blot analysis. cDNA was synthesised by reverse transcription of $4 \mu \mathrm{g}$ of total RNA from each cell line, using an adapter ligated random primer (5'-CAC TGG ATC CTT GAG TTG AGG NNN NNN NNN NNN-3'), followed by low stringency PCR amplification using the same adapter (5'-CAC TGG ATC CTT GAG TTG AGG-3') for priming. PCR products were separated on a $2 \%$ agarose gel and transferred to a GeneScreen Plus ${ }^{\mathrm{R}}$ hybridisation membrane (NEN Research Products, Boston, Massachusetts, USA). Hybridisation was performed using essentially the same conditions as described for northern blot analysis. To confirm equal cDNA loading for each cell line, ethidium bromide staining of the gel was performed and, in addition, membranes were subsequently hybridised with a GAPDH probe.

\section{Results}

mRNA FINGERPRINTS

The repertoire of genes expressed in the GRU-1A and GRU-1B cell lines was compared. Using a set of 90 primer combination, 25 differences in the mRNA fingerprints were observed between the clonal human epithelioid sarcoma cell lines GRU-1A and GRU-1B. Of these, 11 bands were exclusively detected in the highly invasive cell line GRU-1A, whereas 14 bands were found exclusively in the low invasive cell line GRU-1B (fig 1). Despite repetitive efforts, including the use of alternative polymerases and purification of the eluates, only 20 of 25 PCR products could successfully be reamplified after recovering from the gel and were subjected to cloning procedures. Failure to recover the missing five 


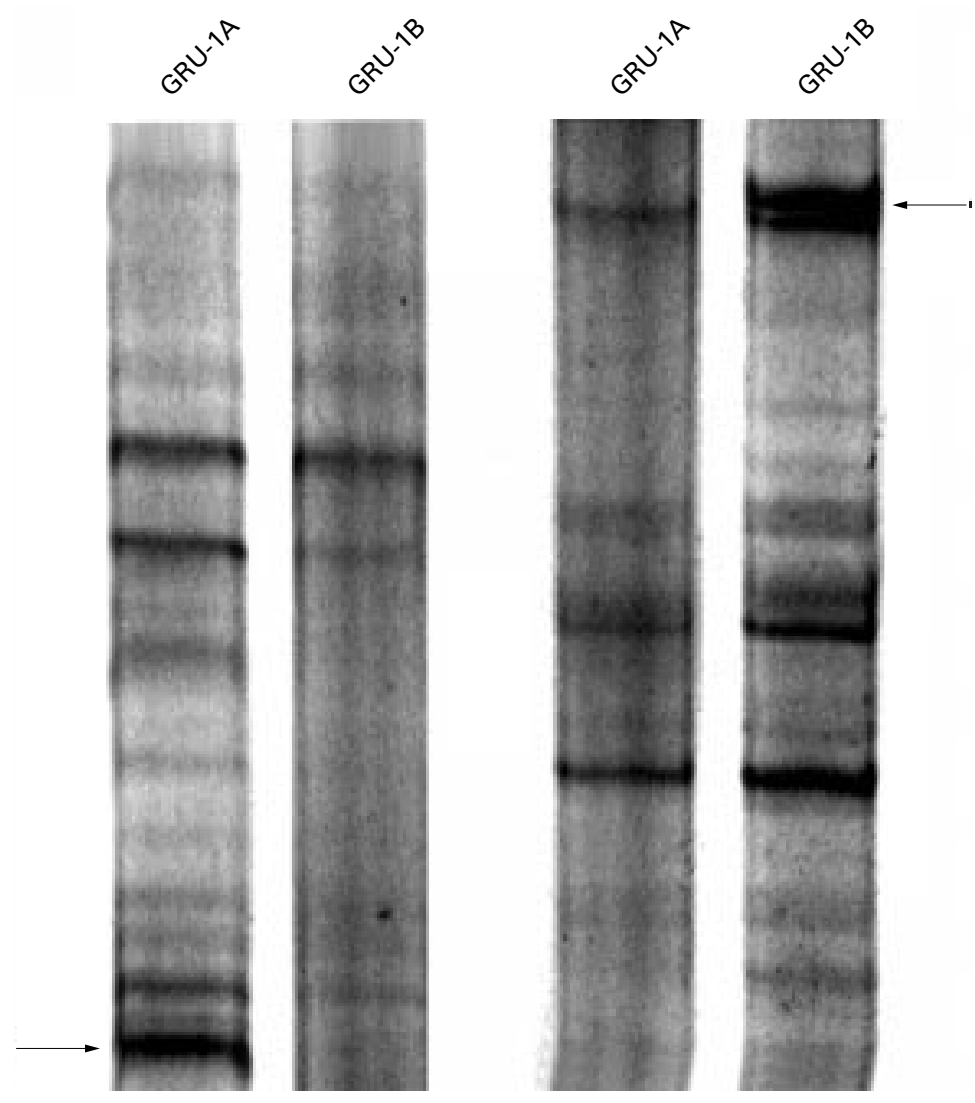

Figure 1 mRNA fingerprinting of GRU-1A and GRU-1B cells. First strand synthesis was performed with Moloney murine leukaemia virus reverse transcriptase using $2 \mu \mathrm{g}$ of total RNA from both cell lines. PCR fingerprinting reactions were performed using pairwise combinations of 10 arbitrary primers $(25 \mathrm{mer}$ ) and nine anchored oligo-dT primers (30 mer). PCR products were run on a denaturing $6 \%$ polyacrylamide gel and visualised by standard silver staining. Examples of bands differentially expressed between the highly invasive cell line GRU-1A and the low invasive cell line GRU-1B are indicated by arrows.

bands is probably the result of interfering components in the gel, as suggested by Konecny and Redinbaugh. ${ }^{18}$
SUBCLONING AND SEQUENCE ANALYSIS

Because a high rate of false positive bands is a well known drawback of differential display screening, ${ }^{19}{ }^{20}$ at least eight white colonies for each transformation procedure were picked to prevent missing the correct insert. A total number of $160(20 \times 8)$ clones was subjected to sequence analysis. Subsequently, clones were preselected for further investigation according to their redundancy and origin of the gene, thus excluding gene fragments encoding rRNA (16 clones from two bands) from further analysis. Table 1 summarises those gene fragments that because of their redundancy were regarded as representative for the respective bands. The mean number of different inserts for each cloning procedure performed for each band was 2.8, ranging from 1 to 4 . Two gene fragments (27.1 and 28.1) originated from rRNA and therefore were excluded from further analysis. Thus, 20 independent clones, recovered from 18 different bands, were subjected to northern blot analysis or Southern blot analysis. Seven of these 22 gene fragments showed no significant homology to currently registered genes in the GenBank databases (table 1).

NORTHERN AND SOUTHERN BLOT ANALYSIS

To verify the differential expression of the gene fragments isolated by fingerprinting, northern and cDNA Southern blot procedures were performed as independent methods. Thus, we were able to detect signals in a total of nine blotting procedures. In contrast, for 13 gene fragments signals were not detectable despite repetitive attempts. We attribute this either to very low expression of the corresponding genes or to the shortness of the respective probes used, or a combination of both. At the mRNA level, no differences in expression between GRU-1A and GRU-1B were seen for five of the nine gene fragments that yielded a useful signal. However, four genes were preferentially

Table 1 Sequence analysis of the isolated cDNA clones and comparison with the GenBank database

\begin{tabular}{llllll}
\hline Clone & Code & Cell line & Blast search & Homology & Blot \\
\hline 1 & 3.1 & B & Human bac clone GS310A05 from 7q21 & $71 \%$ & NA \\
2 & 4.1 & A & Human v-fos effector protein & $100 \%$ & ND \\
3 & 5.1 & B & None, putative novel fragment & - & NA \\
4 & 6.1 & B & None, putative novel fragment & - & NA \\
5 & 9.1 & A & None, putative novel fragment & NA \\
6 & 12.1 & B & Human mitochondrial genes for several t-RNAs and 12S and & $97 \%$ & ND \\
7 & 13.1 & B & 16S ribosomal RNAs & - & B \\
8 & 14.1 & B & Hume, putative novel fragment & $100 \%$ & NA \\
9 & 19.1 & B & Human mitochondrial DNA, fragment M1, encoding & $98 \%$ & B \\
& & & cytochrome C oxidase I & $100 \%$ & NA \\
10 & 22.2 & A & Human SREBP-1 & - & NA \\
11 & 22.4 & A & None, putative novel fragment & $97 \%$ & NA \\
12 & 25.2 & B & None, putative novel fragment & $100 \%$ & ND \\
13 & 26.1 & B & Type VI collagen alpha 3 chain & $100 \%$ & NP \\
14 & 27.1 & A & Human 28S ribosomal RNA & $97 \%$ & NP \\
15 & 28.1 & B & Human 28S ribosomal RNA & - & NA \\
16 & 29.2 & A & PEG3 & $93 \%$ & NA \\
17 & 36.1 & A & None, putative novel fragment & $100 \%$ & B \\
18 & 42.1 & A & Human chromosome 16p13.11 bac clone CIT987SK-29B12 & $100 \%$ & A \\
19 & 43.2 & B & TI-227H & $91 \%$ & NA \\
20 & 51.1 & A & Human ferritin light chain & $100 \%$ & ND \\
21 & 13.2 & B & Human mitochondrion & Human hepatitis $\delta$ interacting protein A & \\
22 & 26.4 & A & &
\end{tabular}

Cell line refers to the clonal cell line from which the cDNA clones were amplified: A, GRU-1A; B, GRU-1B.

Blot refers to results obtained by northern blot analysis: ND, no difference in expression; NA, no blot result available; NP, no blot performed; A, predominantly expressed in GRU-1A; B, predominantly expressed in GRU-1B. 
expressed by only one of the two cell lines (fig 2 ). For clones designated as 13.1, 19.1, and 43.2, significantly stronger expression was seen in the low invasive cell line GRU-1B than in the highly invasive cell line GRU-1A. In contrast, clone 51.1 was significantly more highly expressed by the GRU-1A cell line. Figure 3 gives the nucleotide sequences of gene fragments that were differentially expressed between the two cell lines. Differences in gene expression observed by northern blotting were confirmed by independent controls with additional blotting procedures, using RNA preparations of cells of a higher passage number (data not shown). In all cases, results were reproducible, indicating that the observed differences were stably conserved during prolonged cell culturing.

By means of sequence analysis, clone 51.1 was found to be $100 \%$ identical to human apoferritin light chain (BLASTN value, 4.6e-37). Northern blot analysis with the corresponding probe and measurement by densitometry revealed that the mean (SD) expression of this gene was 1.85 (SD, 0.12) times higher in GRU-1A than in GRU-1B (fig 2).

A computer search for clone 19.1 against the GenBank data base revealed that the identified insert of $201 \mathrm{bp}$ was a fragment of human mitochondrial DNA, encoding cytochrome C oxidase I (BLASTN value, 2.8e-66; 100\% identity). By means of northern blot analysis using the corresponding probe, two distinct signals were seen in both cell lines: one of about $1.5 \mathrm{~kb}$ and a second, albeit weaker one, of about $3.5 \mathrm{~kb}$ (fig 2). In contrast to apoferritin light chain, the mean (SD) expression of cytochrome C oxidase I was 3.34 (0.88) times higher in the low invasive cell line GRU-1B than in GRU-1A.

Clone 43.2 was found to be $100 \%$ identical (BLASTN value, 2.0e-29) to TI-227H. TI$227 \mathrm{H}$ was isolated as a novel gene fragment from the mouse melanoma cell line B16-F10 in an attempt to search for metastasis related genes by subtractive hybridisation. ${ }^{21}$ In our study, northern blot analysis revealed a strong signal in GRU-1B cells just below the $18 \mathrm{~S}$ rRNA band, corresponding to mRNA of about $2 \mathrm{~kb}$. By densitometry, the mean (SD) expression of TI-227H in GRU-1B cells proved to be $2.01(0.16)$ times higher than in the GRU-1A cell line (fig 2).

No significant match to known genes in the GenBank database was found for a $207 \mathrm{bp}$ gene fragment (clone 13.1), which is therefore probably a novel gene. Northern blot analysis revealed a single transcript of about $1 \mathrm{~kb}$, the mean (SD) expression of which was 1.66 (0.19) times higher in the low invasive cell line GRU-1B than in the highly invasive cell line GRU-1A (fig 2). Therefore, we tentatively designated this gene as expressed in low invasive sarcoma cells (ELISC-1). The partial sequence has been released to NCBI GenBank and is available under accession number AF085351. Translation of the $398 \mathrm{bp}$ sequence revealed an open reading frame of 49 amino acids running through the 3' end of this clone. The corresponding predicted amino acid sequence

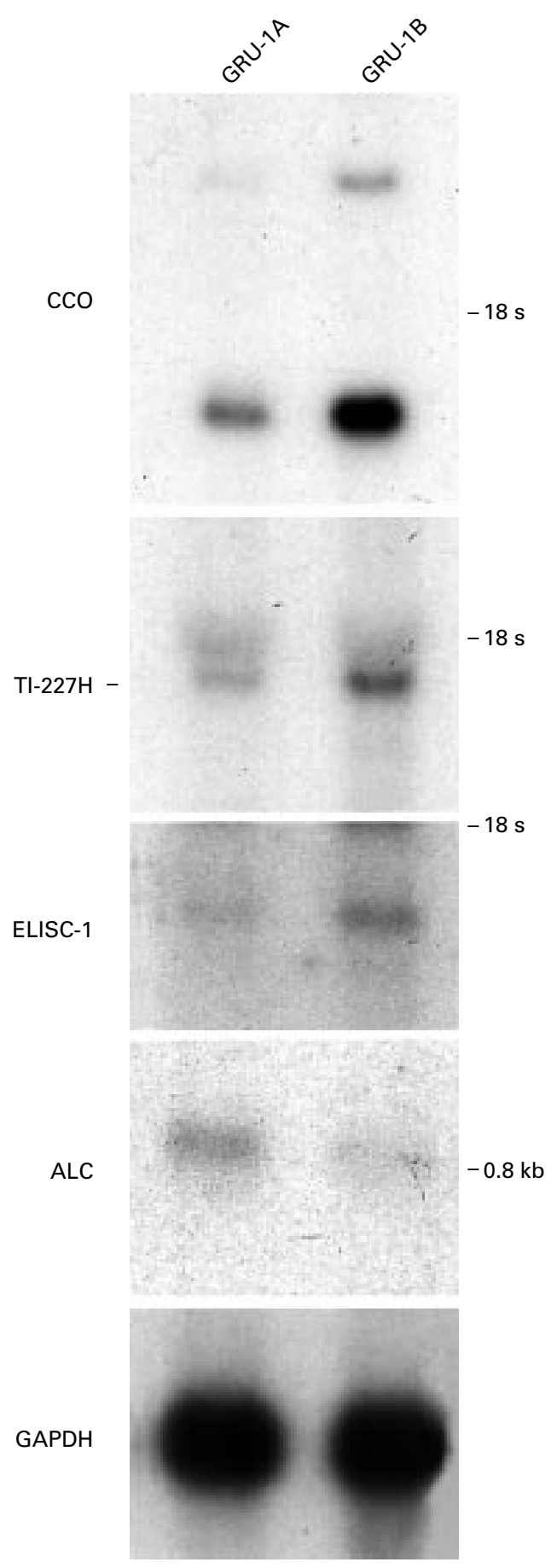

Figure 2 Differential expression of apoferritin light chain (ALC), cytochrome c oxidase I (CCO), TI-227H, and expressed in low invasive sarcoma cells (ELISC-1) between the highly invasive cell line GRU-1A and the low invasive cell line GRU-1B, as shown by northern blot analysis. Aliquots of $15 \mu \mathrm{g}$ of total RNA were separated on a $1 \%$ agarose formaldehyde gel, blotted on to Hybond $N$ nylon agarose formaldehyde gel, blotted on to Hybond N nylon
filters, and probed with DNA probes generated by PCR from the corresponding clone. Equal loading was confirmed by hybridisation with a glyceraldehyde 3-phosphate dehydrogenase (GAPDH) probe. Whereas $A L C$ was preferentially expressed by $G R U-1 A$, the expression of CCO, TI-227, and ELISC-1 was higher in GRU-1B. 
did not show significant homologies to any known protein sequence. While preparing this manuscript, the sequence of ELISC-1 was

Apoferritin light chain

$\begin{array}{rlllll}\frac{1}{51} & \text { ATTAACCCTC } & \text { ACTAAATGCT } & \text { GGGTGGCCCG } & \text { GAGGCTGGGC } & \text { TGGGCGAGTA } \\ \frac{101}{101} & \text { GCGACTTCTG } & \text { AGGCTCACTC } & \text { TCAAGCACGA } & \text { CTAAGAGCCT } & \text { TCTGAGCCCA } \\ \frac{151}{201} & \text { CTCCCTCCAG } & \text { CCAATAGGCA } & \text { TGCAAAGTAA } & \text { TAGGGCTTCT } & \text { GCCTAAGCCTAA } \\ \frac{201}{251} & \text { AATG } & & \text { CTATCCTAAC } & \text { AAGCCTTGGA } \\ & & & & & \end{array}$

ELISC-1

\begin{tabular}{|c|c|c|c|c|c|}
\hline 1 & ATTAACCCTC & ACTAAAGCAC & CGTCCCAGTA & CTCAACTGCT & ATGTAAGAAT \\
\hline $5 \overline{1}$ & $\overline{\text { GCTTTCTTAT }}$ & $\overline{\text { GTGGTAAATG }}$ & TCTCAGTATT & TTGCTGCCTG & GTATTTGTTC \\
\hline $1 \overline{01}$ & AGTTPCCTTG & TATATCTCAG & GGTCAGAAAG & AATCAGGCTT & TCTCCCAACT \\
\hline$\overline{151}$ & CTGAAACATT & CAGACTTACT & TTCTTTTTGG & TCAGCCTTTT & AACAAGCAAG \\
\hline$\frac{101}{201}$ & ACAATAAACT & CCTTTTGTCA & GAATCGATTT & GATTAAAAAA & AAAGATATCA \\
\hline 251 & CTCAGCATAA & $\mathrm{TG}$ & & & \\
\hline
\end{tabular}

Cytochrome $\mathrm{C}$ oxidase 1

\begin{tabular}{|c|c|c|c|c|c|}
\hline 1 & ATTAACCCTC & ACTAAAGCAC & CGTCCCCTGA & ACTCTACACA & ACATATTTTG \\
\hline $5 \overline{1}$ & $\overline{\mathrm{TCACCAAGAC}}$ & $\overline{\text { CCTGACCCTG }}$ & AACICTACAC & AACATATTTT & CAAGA \\
\hline $1 \overline{01}$ & CCCTACTTCT & AACCTCCCTG & TTCTTATGAA & TTCGAACAGC & $\operatorname{CCGA}$ \\
\hline 151 & TTCCGCTACG & ACCAACTCAT & ACACCTCCTA & TGAAAAAACT & דיד \\
\hline 201 & CACCCTAGCA & TTACTTATAT & GATATGTCTC & CATACCCATT & ATO \\
\hline$\overline{251}$ & GCATTCCCCC & TCAAACCGAA & AAAAAAAGAT & $\underline{\mathrm{ATCACTCAGC}}$ & ATAATG \\
\hline
\end{tabular}

TI-227H

$\begin{array}{rlllll}\frac{1}{51} & \text { ATTAACCTCA } & \text { CTAAAGATCT } & \text { GACTGACTGA } & \text { CAATTAACAG } & \text { CCCAATATCT } \\ 1 \frac{1}{01} & \text { ATGCATCAACC } & \text { AACAAGTCATA } & \text { TATTACCCTC } & \text { ACTGTCAACC } & \text { CAACACAGGC } \\ \underline{10} & & \text { GGAAAGGTAA } & \text { AAAAAAAGAT } & \text { ATCACTCAGC } & \text { ATAATG }\end{array}$

Figure 3 Sequences of genes differentially expressed in GRU-1 A and GRU-1 B as confirmed by northern blot analysis. Underlined nucleotides refer to primers used for amplification. Whereas the expression of apoferritin light chain was much stronger in the highly invasive cell line GRU-1A, the expression of expressed in low invasive sarcoma cells (ELISC-1), cytochrome C oxidase I, and TI-227 was higher in the low invasive cell line GRU-1B.

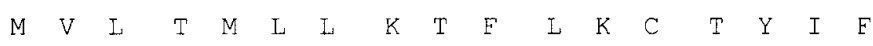

1 aatggttttg actatgcttt taaaacatt tttaaatgt acttacatct

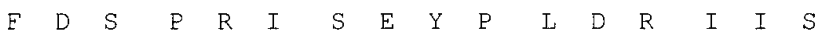

51 tttcgatag cccacgtatt tcagaatatc ctcttgatag aataatatca

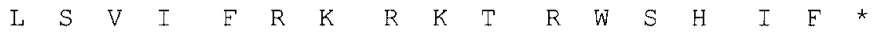

101 ctcagtgtga tttttagaaa aagaaaact cggtggtctc atatcttttg

151 acagttgttt gtgaataata ccctcccaa caaccttcc agtactcaac c agtactcaac

201 tgctatgtaa gaatgcttc ttatgtggta aatgtctcag tatttgctg tgctatgtaa gaatgctttc ttatgtggta aatgtctcag tatttgctg

251 cctggtatt gttcagtttc cttgtatatc tcagggtcag aagaatcag cctggtattt gttcagtttc cttgtatatc tcagggtcag aaagatcag

301 getttctccc aactctgaaa cattcagact tacttettt ttggtcagcc gcttctccc aactctgaaa cattcagact tactttcttt ttggtcagcc

351 ttttaacaag caagacaata aactcctttt gtcagaatcg atttgatt ttttaacaag caagacala aactccttt gtcagaatcg atttga

Figure 4 Alignment of the partial nucleotide sequence of expressed in low invasive sarcoma cells (ELISC-1) (lower lanes), identified in our study, and a known human cDNA clone of 398 bp in the GenBank expressed sequence tag (EST) database (upper lanes) (accession number AA975427). Translation of the sequence revealed that this clone probably harbours the 3' end of the human $c D N A$ sequence of ELISC-1 and the adjacent 3' untranslated region. Further analysis of the 398 bp sequence revealed no significant homologies to any known gene or any other sequence in EST databases. identified as being identical to a part of human BAC clone RP11-288C18 (accession number AC007382), which harbours the striatin gene, a calmodulin binding protein, preferentially expressed in the brain. ${ }^{22}{ }^{23}$ In addition, a search against expressed sequence tag (EST) databases revealed a partial identity to an EST clone (accession number AA975427) (fig 4).

\section{Discussion}

In our present study, mRNA differential display was applied with the objective of identifying genes associated with the different invasive potentials of two clonal subpopulations, derived from the same human epithelioid sarcoma cell line GRU-1. Four genes were found to be differentially expressed between highly invasive GRU-1A and low invasive GRU-1B cells. By means of sequence analysis, three gene fragments proved to be homologous to known genes, and one was novel. This last gene was preferentially expressed in the low invasive cell line GRU-1B and therefore designated as expressed in low invasive sarcoma cells (ELISC-1). Northern blot analysis revealed a single transcript of about $1 \mathrm{~kb}$. While preparing this manuscript, the sequence of ELISC-1 was identified as being identical to a part of human BAC clone RP11-288C18 (accession number AC007382). According to annotated features of this clone, the ELISC-1 sequence is part of the 3 ' untranslated region of the human striatin gene. However, our analysis revealed an open reading frame for the entire length of our fragment. Therefore, it is also possible that the observed differences in transcription of ELISC-1 represent differences in the expression of striatin or a spliced variant thereof. In addition, a search against EST databases revealed a partial identity to an EST clone (accession number AA975427). At present, it remains to be determined whether the ELISC-1 fragment is part of the striatin gene or represents a distinct gene. Although a role for ELISC-1 or striatin in invasion is unknown, its higher expression in clonal sarcoma cells with a low invasive potential suggests that it might play an inhibitory role.

Of the remaining three genes identified, apoferritin light chain was strongly expressed in GRU-1A, but could hardly be detected in GRU-1B (fig 2). Although investigations on the role of apoferritin light chain in tumour invasiveness have not been reported, using subtractive hybridisation (a technique related to differential display RT-PCR), another group found that the expression of a partial sequence of ferritin light chain correlated with differences in the metastasising behaviour of mouse melanoma cells. ${ }^{21}$ However, they could not confirm their results by independent blotting analysis. Nevertheless, increased expression of ferritin mRNA has also been linked to the metastatic potential of other cell types. It has been reported that metastatic ovarian tumour cells express significantly higher amounts of ferritin heavy chain compared with the corresponding primary tumours. ${ }^{24}$ The fact that increased expression of ferritin was seen in tumour metastases of different histogenic 
origin suggests that increased expression of ferritin is a more general feature associated with the metastatic phenotype of tumour cells. In addition, it has been suggested that increased storage of total ferritin occurs in parallel with the development of hyper-resistance to photodynamic inactivation and $\mathrm{H}_{2} \mathrm{O}_{2}$ mediated cytotoxicity of tumour cells, ${ }^{25}$ probably by acting as an intracellular scavenger for toxic iron metabolites. Taking these observations into account, one might speculate that high concentrations of ferritin provide an advantage for metastasising cancer cells in adopting a foreign environment.

A third gene that was differentially expressed between GRU-1A and GRU-1B was the mitochondrial M1 gene, encoding cytochrome c oxidase I. This gene was much more highly expressed in the low invasive cell line GRU-1B. Interestingly, a strong positive correlation between increased expression of mitochondrial genes and the grade of differentiation has been demonstrated for a variety of tumour and nontumour cells of different histogenic origin - for example, HT-29 colon carcinoma cells and human fibroblasts. ${ }^{27}{ }^{28}$ We have shown previously that GRU-1B cells have a predominantly mesenchymal phenotype, whereas GRU-1A cells have a bidirectional differentiation pattern, which confers a combined mesenchymalneural phenotype. ${ }^{8}$ It remains to be determined whether the known interclonal differences in differentiation and invasion are causally related to the newly identified difference in expression of cytochrome C oxidase I.

Finally, in the low invasive GRU-1B cell line we also found preferential expression of a gene that was identical to TI-227H. TI-227H was originally identified by subtractive hybridisation as a gene fragment that, at the mRNA level, was differentially expressed in variant mouse melanoma cell lines of the same primary tumour, but with different metastatic capacities. $^{21}$ In this study, TI-227H was highly expressed in B16-F10 melanoma cells, which can only colonise the lung when directly inoculated into the blood vessels. In contrast, TI-227H was absent in B16-BL-6 cells, which are capable of spontaneously metastasising to the lung upon subcutaneous implantation. Because metastasis is initiated by the process of invasion, both these and our results suggest that TI-227H might have an invasion inhibitory function. When TI-227H was identified, no significant matches with known genes were found. In contrast, our search revealed significant homologies to mitochondrial genes for several t-RNAs (accession number V00710). This would explain the lack of an open reading frame for $\mathrm{TI}-227 \mathrm{H}$ described by Ishiguro et $a l,{ }^{21}$ and indicate a role at least in expression control. However, an RNA signal of about $2 \mathrm{~kb}$ seen for TI-227H by northern blotting using total RNA as a template is unusual for t-RNA. Although these conflicting results cannot be explained at present, it is remarkable that, while searching for invasion or metastasis related genes, TI-227H was independently isolated by means of two different technical approaches (differential display RT-PCR versus subtractive hybridisation) and in two different tumour models (human sarcoma versus mouse melanoma). This strongly suggests a potential role for $\mathrm{TI}-227 \mathrm{H}$ in tumour invasion and metastasis.

When compared with other studies using differential display RT-PCR, both the total number of bands, differentially expressed in fingerprinting reactions, and the rate of real differences in expression as confirmed by independent blotting procedures (five of nine blotting procedures) are similar. ${ }^{29} 30$ Moreover, similar rates of non-informative blotting procedures have also been reported by several other groups. ${ }^{31}{ }^{32}$ Despite repetitive attempts, in our present study, no interpretable signals could be obtained by northern blot analysis for a total number of 11 candidate gene fragments. This is probably the result of the scarcity of the respective mRNAs or suboptimal hybridisation procedures because these problems were only seen for the shortest fragments. ${ }^{29} 3132$ In an attempt to overcome this problem, Southern blot analyses at the cDNA level were performed. Again, signals obtained by Southern blot analysis were only seen for gene fragments that had already been detected by northern blot analysis.

In conclusion, we have identified three known genes (apoferritin light chain, cytochrome $\mathrm{C}$ oxidase I, and $\mathrm{TI}-227 \mathrm{H}$ ) and one novel gene (ELISC-1) that are differentially expressed between highly invasive GRU-1A and low invasive GRU-1B cells. Identification of the entire cDNA sequence of ELISC-1 and analysis of the role of all four genes in the regulation of invasiveness will be the subject of further investigations.

We thank Mrs S Sliwka, Mrs A Florange-Heinrichs, and Mr M Ringler for their excellent technical assistance and Dr BM Ghadimi for helpful discussions and critically reviewing the manuscript.

1 Mareel MM, van Roy FM, Bracke ME. How and when do tumor cells metastasize? Crit Rev Oncog 1993;4:559-94

2 Aznavoorian S, Murphy AN, Stetler-Stevenson WG, et al. Molecular aspects of tumor cell invasion and metastasis. Cancer 1993;71:1368-83.

3 Gabbert H. Mechanisms of tumor invasion: evidence from in vivo observations. Cancer Metastasis Rev 1985;4:293309.

4 Fidler IJ, Radinsky R. Genetic control of cancer metastasis [editorial]. F Natl Cancer Inst 1990;82:166-8.

5 Engers R, Gabbert HE. Mechanisms of tumor metastasis: cell biological aspects and clinical implications. $f$ Cancer Res Clin Oncol 2000;126:682-92.

6 Gerharz CD, Moll R, Ramp U, et al. Multidirectional differentiation in a newly established human epithelioid sarcoma cell line (GRU-1) with co-expression of vimentin, cytokeratins and neurofilament proteins. Int f Cancer 1990;45: eratins and

7 Engers R, Gerharz CD, Donner A, et al. In vitro invasiveness of human epithelioid-sarcoma cell lines: association with cell motility and inverse correlation with the expression of tissue inhibitor of metalloproteinases. Int f Cancer 1999;80: 406-12.

8 Engers R, Gerharz CD, Moll R, et al. Interclonal heterogeneity in a human epithelioid-sarcoma cell line (GRU-1). Int f Cancer 1994;59:548-53.

9 Engers R, van Roy F, Heymer T, et al. Growth inhibition in clonal subpopulations of a human epithelioid sarcoma cell line by retinoic acid and tumour necrosis factor alpha. $B r F$ Cancer 1996;73:491-8.

10 Sager R. Expression genetics in cancer: shifting the focus from DNA to RNA. Proc Natl Acad Sci $U S A$ 1997;94:952-5.

11 Liang P, Pardee AB. Differential display of eukaryotic messenger RNA by means of the polymerase chain reaction [see comments]. Science 1992;257:967-71.

12 Yu Y, Xu F, Peng H, et al. NOEY2 (ARHI), an imprinted putative tumor suppressor gene in ovarian and breast carcinomas. Proc Natl Acad Sci US A 1999;96:214-19. 
13 Fournier MV, Guimaraes FC, Paschoal MM, et al. Identification of a gene encoding a human oxysterol-binding protein-homologue: a potential general molecular marker for blood dissemination of solid tumors. Cancer Res 1999;59:3748-53.

14 Wan JS, Sharp SJ, Poirier GM, et al. Cloning differentially expressed mRNAs. Nat Biotechnol 1996;14:1685-91.

15 Bassam BJ, Caetano AG, Gresshoff PM. Fast and sensitive silver staining of DNA in polyacrylamide gels [published erratum appears in Anal Biochem 1991;198:217]. Anal Biochem 1991;196:80-3.

16 Feinberg AP, Vogelstein B. A technique for radiolabeling DNA restriction endonuclease fragments to high specific activity. Anal Biochem 1983;132:6-13.

17 Feinberg AP, Vogelstein B. "A technique for radiolabeling DNA restriction endonuclease fragments to high specific activity". Addendum. Anal Biochem 1984;137:266-7.

18 Konecny P, Redinbaugh MG. Amplification of differentially displayed PCR products isolated from untreated denaturing polyacrylamide gels. Biotechniques 1997;22:240-4.

19 Miele G, MacRae L, McBride D, et al. Elimination of false positives generated through PCR re-amplification of differpositives generated through PCR re-amplification of
ential display cDNA. Biotechniques 1998;25:138-44.

20 Luce MJ, Burrows PD. Minimizing false positives in differential display. Biotechniques 1998;24:766-70.

21 Ishiguro T, Nakajima M, Naito M, et al. Identification of genes differentially expressed in B16 murine melanoma sublines with different metastatic potentials. Cancer Res 1996;56:875-9.

22 Castets F, Bartoli M, Barnier JV, et al. A novel calmodulinbinding protein, belonging to the WD-repeat family, is localized in dendrites of a subset of CNS neurons. $\mathcal{F} \mathrm{Cell}$ Biol 1996;134:1051-62.

23 Castets F, Rakitina T, Gaillard S, et al. Zinedin, SG2NA, and striatin are calmodulin-binding, WD repeat proteins principally expressed in the brain. $\mathcal{F}$ Biol Chem $2000 ; 275: 19970-7$.
24 Tripathi PK, Chatterjee SK. Elevated expression of ferritin H-chain mRNA in metastatic ovarian tumor [see comments]. Cancer Invest 1996;14:518-26.

25 Lin F, Girotti AW. Hyperresistance of leukemia cells to photodynamic inactivation after long-term exposure to hemin. Cancer Res 1996;56:4636-43.

26 Cermak J, Balla J, Jacob HS, et al. Tumor cell heme uptake induces ferritin synthesis resulting in altered oxidant sensitivity: possible role in chemotherapy efficacy. Cancer Res 1993;53:5308-13.

27 Corral M, Paris B, Baffet G, et al. Increased level of the mitochondrial ND5 transcript in chemically induced rat hepatomas. Exp Cell Res 1989;184:158-66.

28 Torroni A, Stepien G, Hodge JA, et al. Neoplastic transformation is associated with coordinate induction of nuclear and cytoplasmic oxidative phosphorylation genes. $\mathcal{F}$ Biol Chem 1990;265:20589-93

29 Liang P, Averboukh L, Pardee AB. Distribution and cloning of eukaryotic mRNAs by means of differential display: refinements and optimization. Nucleic Acids Res 1993;21: refinements

30 Lu X, Seligy VL. Mitochondrial RNA abundance in differentiating human colonic epithelial tumor cells estimated through use of a mitochondrial genome map. Gene 1993;131:217-25

31 Liang P, Averboukh L, Keyomarsi K, et al. Differential display and cloning of messenger RNAs from human breast cancer versus mammary epithelial cells. Cancer Res 1992;52:6966-8.

32 Hashimoto Y, Shindo ON, Tani M, et al. Identification of genes differentially expressed in association with metastatic potential of K-1735 murine melanoma by messenger RNA differential display. Cancer Res 1996;56:5266-71.

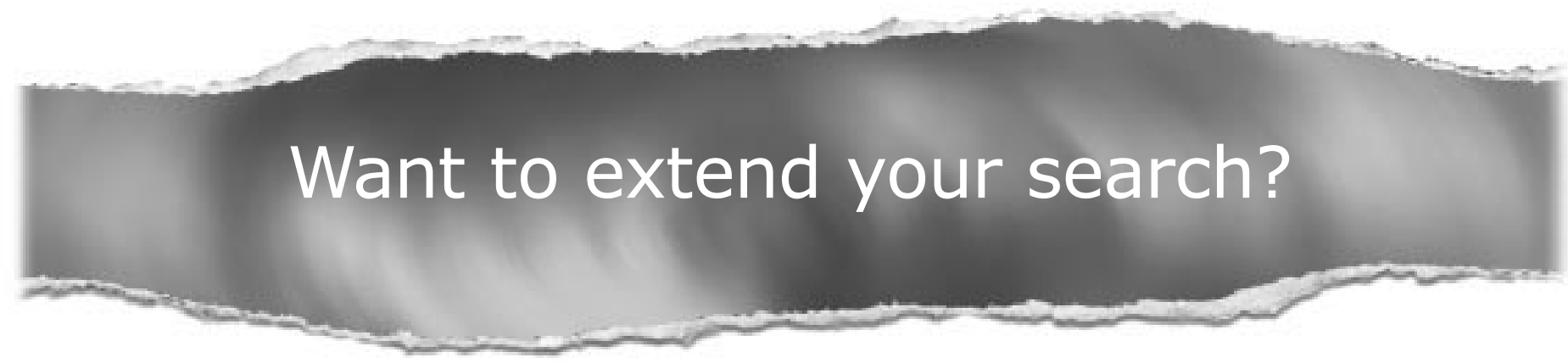

\section{Cross journal searching}

If you can't find what you are looking for in the Journal of Clinical Pathology you can extend your search across many of the more than 200 journals available for selection. You can restrict your search to specific subject areas (eg, clinical medicine, basic research), or select specific journals, or search all available titles.

\section{www.jclinpath.com}

